(2) Open Access Full Text Article

\title{
A primary retroperitoneal anaplastic lymphoma kinase-positive anaplastic large cell lymphoma with tumor thrombosis
}

This article was published in the following Dove Press journal:

OncoTargets and Therapy

\author{
Jisong $\mathrm{Li}^{\prime}$ \\ Xiaodong Tian' \\ Mangju Wang ${ }^{2}$ \\ Wei Liu ${ }^{2}$ \\ Xiaochao Guo ${ }^{3}$ \\ Ke Wang ${ }^{3}$ \\ Lin Nong ${ }^{4}$ \\ Wei Wang ${ }^{4}$ \\ Yinmo Yang' \\ 'Department of General Surgery, \\ Peking University First Hospital, \\ Beijing, China; ${ }^{2}$ Department of \\ Hematology, Peking University First \\ Hospital, Beijing, China; ${ }^{3}$ Department \\ of Radiology, Peking University First \\ Hospital, Beijing, China; ${ }^{4}$ Department \\ of Pathology, Peking University First \\ Hospital, Beijing, China
}

\begin{abstract}
Anaplastic lymphoma kinase (ALK)-positive anaplastic large cell lymphoma (ALCL) is a T cell subtype of non-Hodgkin's lymphoma (NHL). Typically, lymphoma rarely infiltrates vascular structure. In this article, we present a case of retroperitoneal ALK-positive ALCL with splenic venous tumor thrombosis. A 62-year-old patient presented to our institute with the symptoms of epigastric pain, abdominal distension, and reduced bowel movement. Physical examination indicated no enlarged peripheral lymph nodes or abdominal mass. Laboratory workup revealed granulocytosis, abnormal coagulation function, and normal level of lactic dehydrogenase (LDH). Contrast-enhanced computed tomography (CT) showed a retroperitoneal mass with involvement of pancreas and duodenum and formation of splenic venous tumor thrombus. Ultrasonography-guided retroperitoneal lesion biopsy confirmed the diagnosis of ALK-positive ALCL. The patient was able to tolerate oral intake after two cycles of chemotherapy and showed no sign of lymphoma by positron emission tomography (PET)-CT after the fourth cycle of chemotherapy. In spite of its rarity, lymphoma should be taken into account as a differential diagnosis of other malignancies with tumor thrombosis.
\end{abstract}

Keywords: anaplastic lymphoma kinase, anaplastic large cell lymphoma, primary retroperitoneal lymphoma, tumor thrombosis

\section{Introduction}

Anaplastic large cell lymphoma (ALCL) is a subtype of non-Hodgkin's lymphoma (NHL), which derives from cytotoxic T cell phenotype. ${ }^{1}$ Anaplastic lymphoma kinase (ALK)-positive ALCL harbors an ALK-fusion gene, typically via $\mathrm{t}(2 ; 5)(\mathrm{p} 23 ; 35)$, resulting in an NPM-ALK chimeric protein with constitutive tyrosine kinase activity. ${ }^{2}$ ALK-positive ALCL constitutes about 3\% of adult NHL, and nodal and extranodal manifestations are commonly seen. ${ }^{3}$ Lymphoma often encompasses vessels, but seldom infiltrates them, known as the "sandwich" sign or "computed tomography (CT) angiogram" sign. ${ }^{1-3}$ Tumor thrombosis is an extremely rare presentation in lymphoma. ${ }^{3}$ In this article, we present a case of retroperitoneal ALK-positive ALCL causing duodenal stenosis, with tumor thrombus in splenic vein.

\section{Case presentation}

This study was approved by the ethics committee of Peking University First Hospital. Written informed consent has been provided by the patient to have the case details and any accompanying images published. A 62-year-old male patient presented to the local hospital with a 1-month history of epigastric pain, abdominal distension, 
and reduced bowel movement. He denied any fever or drenching night sweats. Abdominopelvic CT scan revealed a retroperitoneal mass beneath the body of pancreas compressing duodenum and the formation of splenic venous tumor thrombus. CT-guided retroperitoneal lesion biopsy was performed, and pathological analysis revealed the possibility of malignant tumor. He was inserted with a nasogastric tube and supported by parenteral nutrition. For further treatment, he was transferred to our tertiary care center. He had a past history of diabetes mellitus type II managed by diet and exercise and spinal dural arteriovenous fistulectomy. Physical examination revealed normal vital signs, no peripheral lymph node enlargement, or palpable abdominal mass.

Laboratory tests showed a striking abnormality of white

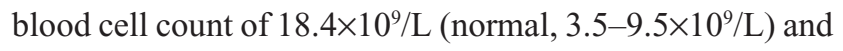
neutrophil cell count of $15.80 \times 10^{9} / \mathrm{L}$ (normal, $1.8-6.3 \times 10^{9} / \mathrm{L}$ ). Coagulation abnormality was obvious as prothrombin time (PT) was prolonged by 7.1 seconds and international normalized ratio (INR) approached 1.81 (normal, 0.89-1.13). Erythrocyte sedimentation rate (ESR) was $80 \mathrm{~mm} / \mathrm{h}$ (normal, 0-15 mm/h). Lactic dehydrogenase (LDH), CA19-9, and CA242 were normal, while CA125 was slightly elevated (47.27 U/mL; normal, <35.0 U/mL).

Abdominopelvic contrast-enhanced CT was suggestive of an amorphous conglomerated hypoattenuating mass underneath the body of pancreas compressed and infiltrated the third segment of duodenum. Splenic vein was not visible, and a longitudinal mildly enhanced mass was seen along and behind the body and tail of pancreas, clotting the superior mesenteric vein-portal vein (SMV-PV) confluence. Lymph nodes at the root of the mesentery and retroperitoneal region enlarged, and some were fused into bulky masses with indistinctive boundaries with aorta (Figure 1).

As the coagulation function improved by the supplement of vitamin $\mathrm{K}$, the patient underwent a second percutaneous core needle biopsy of the retroperitoneal tumor guided by ultrasonography. The pathological analysis showed atypical tumor cells with ovoid or irregular (eg, kidney shaped) nuclei packed in sheets with intrasinusoidal infiltration. Immunohistochemistry (IHC) staining was performed using DAKO EnVison $^{\mathrm{TM}}$ detection kit (Dako Denmark A/S, Glostrup, Denmark) and a panel of antibodies. The percentage of positive cells was semiquantitatively estimated and scored as follows: negative $(-)$ : $<5 \%$ cells; weakly positive $(+): 6 \%-30 \%$ cells; moderately positive $(++): 31 \%-75 \%$ cells; strongly positive $(+++):>75 \%$. IHC analysis showed the following results: $\mathrm{EMA}+++$, vimentin+++, $\mathrm{LCA}++, \mathrm{CD} 43+++, \mathrm{CD} 3-, \mathrm{CD} 20-$, CD79a-, CD30+++, CD68 (KP1)-, CD56+, CD4++, CD8-, TIA1+, granzyme B++, ALK (CD246)-, ALK (1A4)+++ (nuclear/cytoplasmic), Ki67 70\% (Figure 2). Fluorescence in situ hybridization (FISH) detected $A L K(2 \mathrm{p} 23)$ gene rearrangement. The peripheral blood smear and lymphocyte
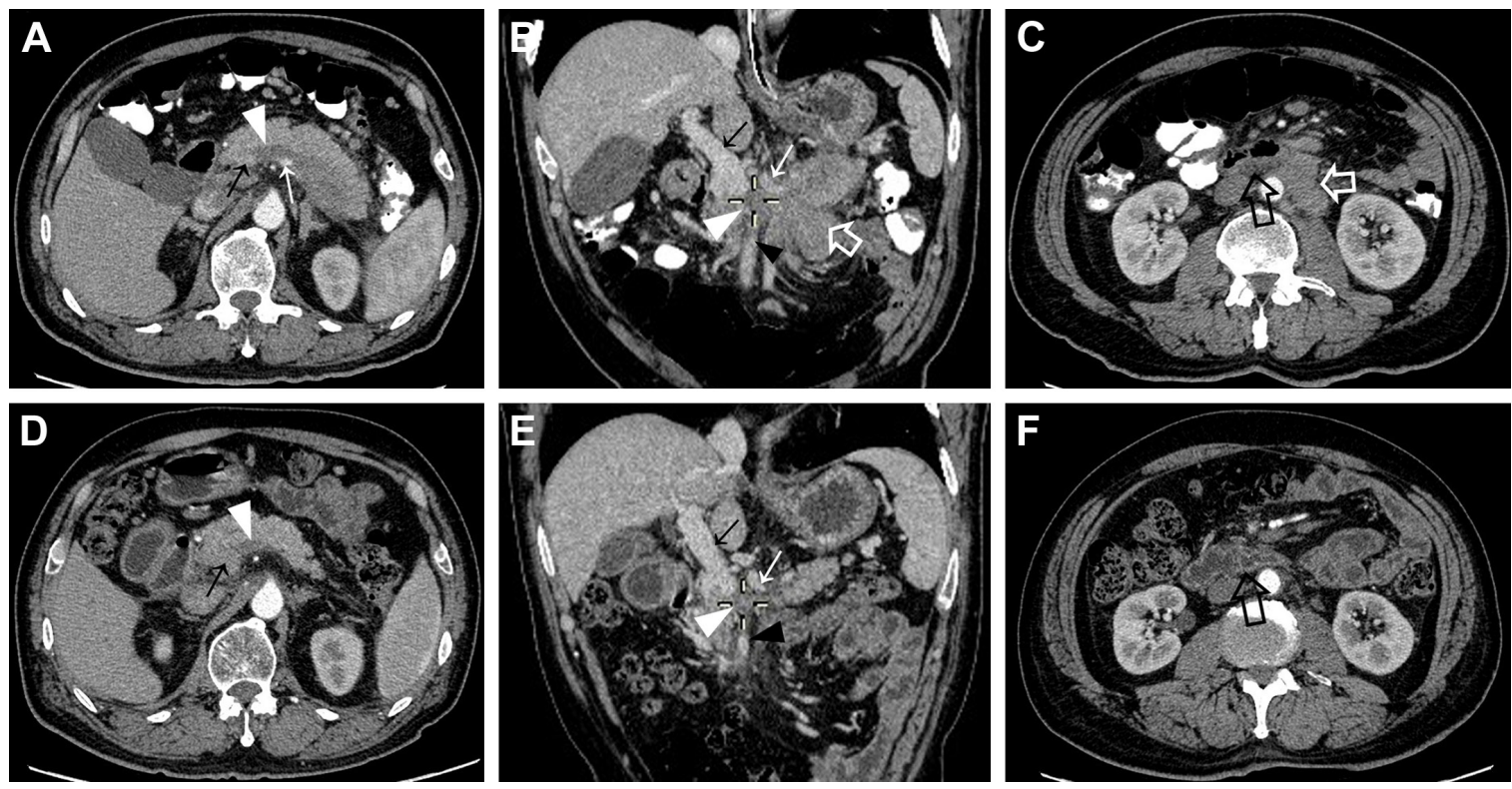

Figure I Abdominopelvic contrast-enhanced CT scan.

Notes: Black arrow: portal vein; white arrow: splenic artery; white arrowhead: splenic venous thrombus; black arrowhead: superior mesenteric vein; white hollow arrow: retroperitoneal lymphoma; black hollow arrow: the third segment of duodenum. (A) Splenic vein was replaced by longitudinal hypoattenuating mass, accompanied with splenic artery. (B) The retroperitoneal mass infiltrated the body of pancreas and splenic vein. The confluences of dilated PSPDV and coronary vein into portal vein could be seen. (C) The retroperitoneal mass involved aorta and posterior wall of duodenum. (D, E) After six cycles of chemotherapy, the splenic thrombus shrank significantly and the retroperitoneal mass disappeared. (F) The para-aorta mass and thickening of duodenum disappeared after the sixth cycle of therapy.

Abbreviations: CT, computed tomography; PSPDV, posterior superior pancreaticoduodenal vein. 

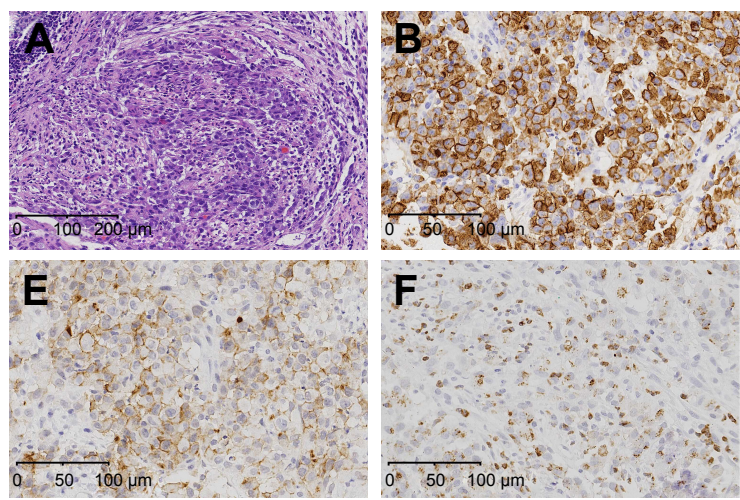
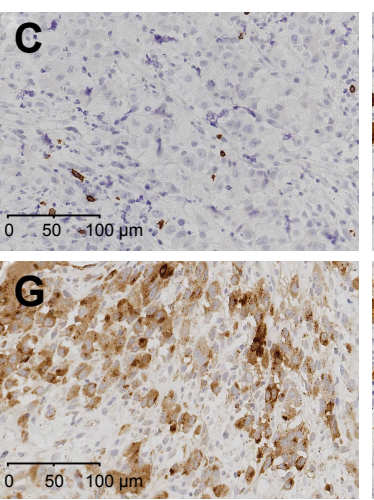
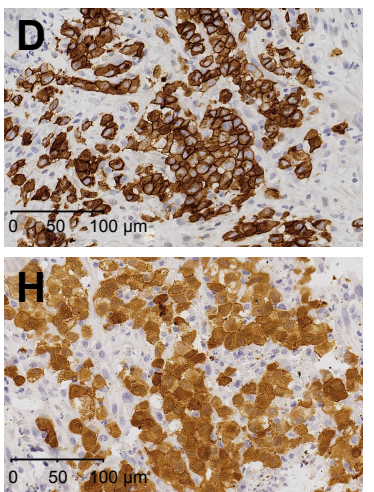

Figure 2 Histopathological and immunohistochemical features of the retroperitoneal mass.

Notes: (A) HE staining of the tumor specimen showed atypical tumor cells with ovoid or irregular nuclei packed in sheets with intrasinusoidal infiltration. Kidney-shaped nuclei and obvious nucleoli were visible in the abundant eosinophilic cytoplasm $(\times 400)$. Mitotic figures could be seen with neutrophil and plasmacyte infiltration. (B) IHC staining of EMA $(\times 200)$. (C) IHC staining of CD3 ( $\times 400)$. (D) IHC staining of CD30 ( $\times 400)$. (E) IHC staining of CD56 ( $\times 400)$. (F) IHC staining of TIAI ( $\times 400)$. (G) IHC staining of granzyme B $(\times 400)$. (H) IHC staining of ALK (IA4) $(\times 400)$. For all antibodies, the percentage of positive cells was semiquantitatively estimated and scored as follows: negative $(-)$ : $<5 \%$ cells; weakly positive $(+): 6 \%-30 \%$ cells; moderately positive $(++): 31 \% \sim 75 \%$ cells; strongly positive $(+++):>75 \%$.

Abbreviation: IHC, immunohistochemistry.

subgroup analysis by FISH revealed no immature cells. Bone marrow smear and bone marrow aspiration revealed no evidence of lymphoma involvement.

Ultrasonography, chest X-ray, and thoracic CT detected neither peripheral nor mediastinal involvement. The final diagnosis was considered as primary retroperitoneal ALCL (stage IV) with duodenal obstruction and splenic venous tumor thrombosis. No source of infection was found in spite of leukocytosis, and broad-spectrum antibiotics had limited effects.
Following the initiation of CHOEP-21 regimen chemotherapy (cyclophosphamide $1.4 \mathrm{~g}$ day (d)1, doxorubicin $40 \mathrm{mg} \mathrm{d} 1$, vincristine $4 \mathrm{mg} \mathrm{d} 1$, etoposide $100 \mathrm{mg} \mathrm{d} 1-\mathrm{d} 3$, and prednisolone $15 \mathrm{mg} \mathrm{d} 1-\mathrm{d} 5$, a cycle contained 21 days, and the patient underwent a total of eight cycles of chemotherapy), leukocyte count decreased significantly and was $4.51 \times 10^{9} / \mathrm{L}$ before the second cycle. The patient was able to tolerate oral intake after the second cycle. Positron emission tomography-CT (PET-CT) showed no sign of lymphoma after the fourth cycle of chemotherapy (Figure 3). A
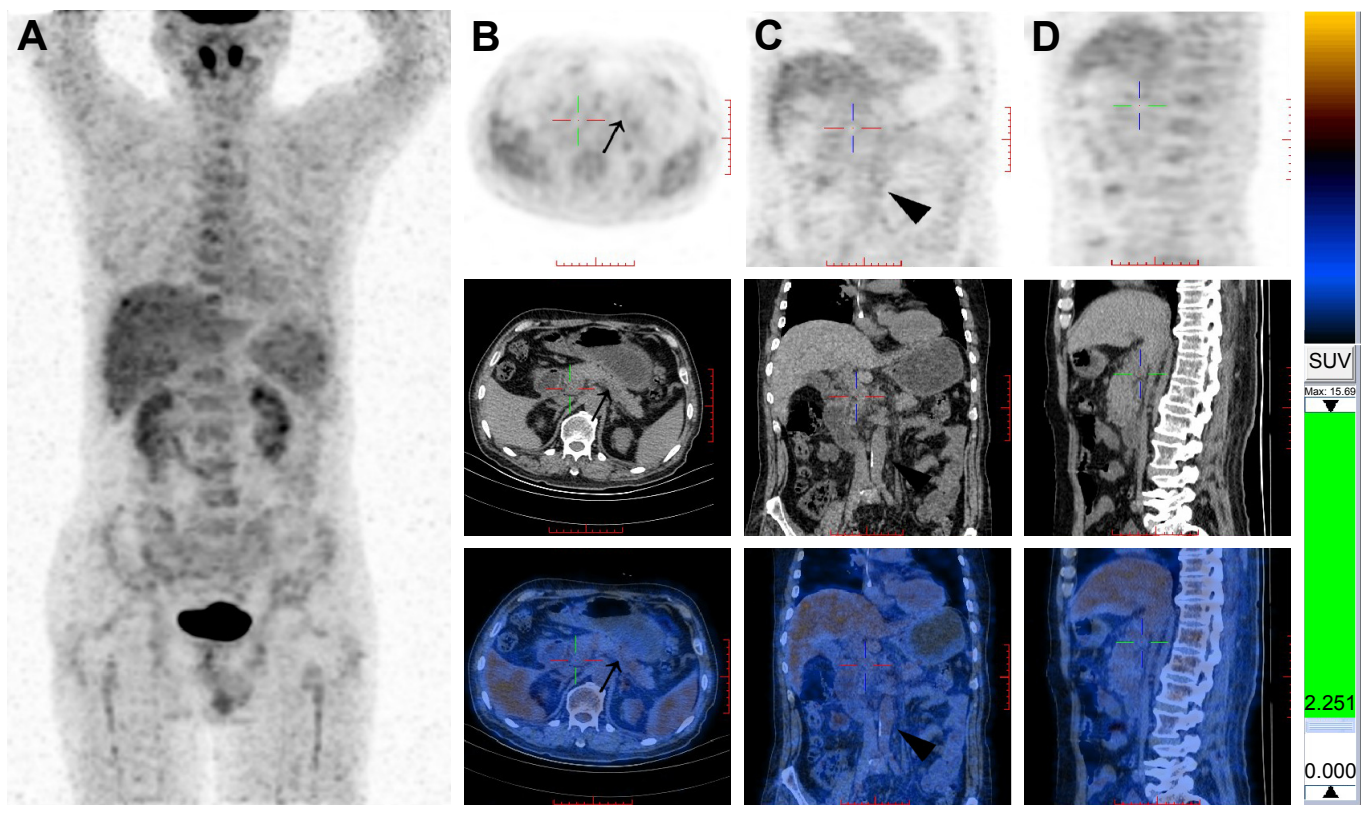

Figure 3 PET-CT.

Notes: (A) No sign of lymphoma was detected after the fourth cycle of chemotherapy. (B) Axial sections revealed normal size pancreas with no elevated intake of FDG (black arrow). (C) Coronal sections showed no peri-aorta lymphadenopathy (black arrowhead) or ascites. (D) Sagittal view showed slightly elevated intake of FDG in spine with normal bone structure, which was presumed as the reactive change after chemotherapy.

Abbreviations: CT, computed tomography; FDG, ${ }^{18} \mathrm{~F}$-fluorodeoxyglucose; PET, positron emission tomography. 
contrast-enhanced CT was repeated after the sixth cycle of chemotherapy, and retroperitoneal masses and lymph nodes disappeared or shrank evidently to normal size compared to the previous CT scan. SMV and splenic veins were still occlusive with slightly enhanced thrombus, but the size of thrombus shrank (Figure 1). The patient was discharged after eight cycles of chemotherapy. He has lived for 4 months with no evidence of recurrence.

\section{Discussion}

ALK-positive ALCL is often at stage III or IV upon diagnosis, with frequent involvement of both lymph nodes and extranodal sites. ${ }^{4}$ In our case, lymphoma extended to retroperitoneal lymph nodes and two extranodal organs, ie, pancreas and duodenum, causing difficulty in differentiating its origin. Primary retroperitoneal lymphoma arises in the retroperitoneal space but outside the major retroperitoneal organs. ${ }^{1}$ Roughly, $55 \%$ of adults with retroperitoneal NHL have involvement of para-aortic lymph nodes. ${ }^{4}$ In our case, the dominant mass was outside pancreas and duodenum and contiguous with para-aorta lymphadenopathy, without peripheral, mediastinal, or bone marrow involvement. Therefore, we postulated that the lesion was primary retroperitoneal lymphoma. It was reported that diffuse pancreatic involvement causes organ enlargement as well as irregular peripancreatic fat infiltration with no other evidence of pancreatitis, ${ }^{5}$ as shown in our case. The pancreas was contiguous with the tumor, both supplied by the dilated dorsal pancreatic artery, the first branch of splenic artery. These signs suggested that the retroperitoneal lymphoma extended to peripancreatic lymph nodes and even the pancreatic parenchyma. Although the patient presented to our department with the symptoms of duodenal obstruction and CT scan showed the thickening of posterior duodenal wall and luminal constriction of third segment of duodenum, the typical sign in primary gastrointestinal lymphoma was circumferential thickening of bowel wall..$^{5}$ It seemed that duodenum was secondarily infiltrated by the compressing retroperitoneal lymphoma rather than a primary lesion.

Lymphoma tends to encase the vascular structure around it but seldom invades..$^{1,3,6}$ Tumor thrombosis is an extremely rare manifestation in lymphoma. As far as we know, only 29 cases of extravascular lymphoma with tumor thrombosis formation have been reported in English literatures. ${ }^{3,7-10}$ The most common pathology is diffuse large B cell lymphoma (DLBCL). Only one case has been reported with the pathology of $\mathrm{T}$ cell lymphoma (mycosis fungoides). ${ }^{3}$ Our case is the first case of ALK-positive ALCL with tumor thrombosis.
The mechanism of the formation of tumor thrombus is mostly presumed as direct extension from a dominant nodal or extranodal mass in the vicinity, ${ }^{3}$ except one reported case of vascular metastasis from splenic lymphoma. ${ }^{8}$ It is important to differentiate tumor thrombosis from primary lymphoma of a vein and intravascular lymphomatosis (IVL). Primary lymphoma of a vein is a highly unusual type of lymphoma centered within a vein, which originates from the vessel wall. ${ }^{11,12}$ However, IVL is a rare type of extranodal large B cell lymphoma characterized by the selective growth within small vessels, particularly capillaries. ${ }^{2}$ In our case, the splenic venous tumor thrombosis had no distinct boundary with the superior border of the dominant extravascular lesion, thus we assumed that lymphoma infiltrated the splenic vein directly. We noticed that the CT value of the thrombus enhanced from the plain phase gradually to the delayed phase at the SMV-PV confluence, whereas the enhancement of thrombus at the tail of pancreas was less obvious. The size of spleen was normal, and pelvic ascites was minimal, probably because of the slow formation of tumor thrombus and the well-developed collateral circulation. ${ }^{13}$ PET-CT showed no sign of lymphoma, but CT revealed that the downsizing thrombus was slightly enhanced after four to six cycles of chemotherapy, which could be attributed to the organization of the mixed thrombus.

Chemotherapy is the major treatment for ALCL at stage III/IV. The initial regimens are recommended as CHOP (cyclophosphamide, doxorubicin, vincristine and prednisone) or CHOEP (cyclophosphamide, doxorubicin, vincristine, etoposide and prednisone). ${ }^{14} \mathrm{LDH}, \mathrm{CD} 56$, and International Prognostic Index (IPI) are useful clinicopathological parameters in predicting outcome. ${ }^{14-16}$ In this case, the patient had positive CD56 expression, but serum LDH was normal. The patient's IPI was 3 (high-intermediate-risk group), with a 5-year overall survival time estimated to $21 \%{ }^{16}$

Since the patient's bone marrow was not involved by lymphoma, we associated the patient's leukocytosis with paraneoplastic leukocytosis. Unlike leukemic phase of lymphoma, the peripheral blood is free of circulating lymphoma cells in paraneoplastic leukocytosis. The mechanism of this phenomenon is the overexpression of cytokines (IL-22, IL-17, G-CSF, TNF- $\alpha$, etc.) in ALK-positive ALCL, facilitating the maturation and proliferation of granulocytes. ${ }^{17}$ Paraneoplastic leukocytosis is postulated as an ominous sign of aggressive clinical course and dismal prognosis. ${ }^{17-19}$ Due to the recent discharge of our patient, further observation and close follow-up are required to monitor his survival. 


\section{Conclusion}

ALCL with tumor thrombosis is rare and warrants biopsy to be differentiated from other malignancies.

\section{Acknowledgment}

This study was supported in part by grants from the National Natural Science Foundation of China (nos. 81372605, 81672353, and 81572339).

\section{Disclosure}

The authors report no conflicts of interest in this work.

\section{References}

1. Rajiah P, Sinha R, Cuevas C, et al. Imaging of uncommon retroperitoneal masses. Radiographics. 2011;31(4):949-976.

2. Sheth S, Horton KM, Garland MR, Fishman EK. Mesenteric neoplasms: CT appearances of primary and secondary tumors and differential diagnosis. Radiographics. 2003;23(2):457535-473456; quiz.

3. Chauhan A, Garg N, Menias CO, et al. Tumor thrombus as a rare presentation of lymphoma: a case series of 14 patients. AJR Am J Roentgenol. 2015;204(4):W398-W404.

4. Neville A, Herts BR. CT characteristics of primary retroperitoneal neoplasms. Crit Rev Comput Tomogr. 2004;45(4):247-270.

5. Leite NP, Kased N, Hanna RF, et al. Cross-sectional imaging of extranodal involvement in abdominopelvic lymphoproliferative malignancies. Radiographics. 2007;27(6):1613-1634.

6. Hokama A, Nakamoto M, Kinjo F, Fujita J. 'The sandwich sign' of mesenteric lymphoma. Eur J Haematol. 2006;77(4):363-364.

7. Natsuizaka M, Kudo M, Suzuki M, et al. Diffuse large B-cell lymphoma with massive portal vein tumor thrombosis in a patient with alcoholic cirrhosis: a case report and literature review. Intern Med. 2009;48(10): 805-808.
8. Ishiguro T, Takayanagi N, Baba $\mathrm{Y}$, et al. Case series of pulmonary tumor embolism and intravascular lymphoma: evaluation of the usefulness of pulmonary microvascular cytology. Intern Med. 2016;55(18): 2679-2684.

9. Chen L, Richendollar B, Bunting S, Campbell S, Zhou M. Lymphomas and lymphoproliferative disorders clinically presenting as renal carcinoma: a clinicopathological study of 14 cases. Pathology. 2013;45(7): 657-663.

10. Samlowski EE, Dechet C, Weissman A, Samlowski WE. Large cell nonHodgkin's lymphoma masquerading as renal carcinoma with inferior vena cava thrombosis: a case report. J Med Case Rep. 2011;5:245.

11. Chauhan A. Reply to "Intravascular Lymphoma". AJR Am J Roentgenol. 2015;205(3):W388.

12. Rulli F, Neri A, Bigotti G, et al. Primary malignant lymphoma of the saphenous vein. J Vasc Surg. 2002;35(1):168-171.

13. Matsumoto S, Mori H, Takaki H, et al. Malignant lymphoma with tumor thrombus in the portal venous system. Abdom Imaging. 2004;29(4): 460-462.

14. Eyre TA, Khan D, Hall GW, Collins GP. Anaplastic lymphoma kinase-positive anaplastic large cell lymphoma: current and future perspectives in adult and paediatric disease. Eur J Haematol. 2014; 93(6):455-468.

15. Sibon D, Fournier M, Brière J, et al. Long-term outcome of adults with systemic anaplastic large-cell lymphoma treated within the Groupe d'Etude des Lymphomes de l'Adulte trials. J Clin Oncol. 2012;30(32): 3939-3946.

16. Sonnen R, Schmidt WP, Müller-Hermelink HK, Schmitz N. The International Prognostic Index determines the outcome of patients with nodal mature T-cell lymphomas. Br J Haematol. 2005;129(3): 366-372.

17. Chang IW, Chen HK, Ma MC, Huang WT, Mc M. Anaplastic large cell lymphoma with paraneoplastic leukocytosis: a clinicopathological analysis of five cases. APMIS. 2011;119(11):794-801.

18. El-Osta HE, Salyers WJ, Palko W, et al. Anaplastic large-cell lymphoma with leukemoid reaction. J Clin Oncol. 2008;26(26):4356-4358.

19. Almakdisi T, Massoud S, Makdisi G. Lymphomas and chylous ascites: review of the literature. Oncologist. 2005;10(8):632-635.
OncoTargets and Therapy

\section{Publish your work in this journal}

OncoTargets and Therapy is an international, peer-reviewed, open access journal focusing on the pathological basis of all cancers, potential targets for therapy and treatment protocols employed to improve the management of cancer patients. The journal also focuses on the impact of management programs and new therapeutic agents and protocols on

\section{Dovepress}

patient perspectives such as quality of life, adherence and satisfaction. The manuscript management system is completely online and includes a very quick and fair peer-review system, which is all easy to use. Visit http://www.dovepress.com/testimonials.php to read real quotes from published authors. 